\title{
Impacto económico del XXI Campeonato de Espańa "Open" de Invierno Master de Natación de Pontevedra 2015
}

\section{Economic impact of the XXI Winter Masters Spanish "Open" Swimming Championship in Pontevedra 2015}

\author{
Sánchez Fernández, P., Salgado Barandela, J., Rodríguez Rodríguez, A. y Barajas Alonso, A.
}

Universidad de Vigo (España).

\begin{abstract}
Resumen: El presente artículo surge con el objetivo de analizar los efectos de la celebración de un evento deportivo en una localidad. En concreto, se presta atención al impacto económico de la celebración en el municipio de Pontevedra del XXI Campeonato de España “Open” de Invierno de Natación Master de Natación en febrero de 2015. Paralelamente se analiza el perfil de los asistentes a dicho evento desde un punto de vista del perfil de "turista deportivo" a partir de la realización de una encuesta específica para el evento. Palabras clave: eventos deportivo, impacto, natación, Pontevedra, turismo deportivo
\end{abstract}

Abstract: The aim of this study consists of analyzing the effects of the celebration of a sport event in a location. In particular, attention is paid to the economic impact of the celebration in the municipality of Pontevedra of the XXI Winter Masters Spanish "Open” Swimming Championship in February 2015. Moreover it analyzes the profile of the assistants to this event from a point of view of the "sport tourist" according to a specific survey carried out for the event.

Keywords: sport event, impact, swimming, Pontevedra, sports tourism.

\section{Introducción}

El deporte genera un gran interés social, económico y mediático propiciando así la celebración de eventos deportivos de mayor o menor importancia. Shone y Parry (2001) definen a los eventos como "fenómenos que surgen de ocasiones rutinarias y que tiene objetivos de ocio, culturales, personales u organizativos establecidos de forma separada a la actividad normal diaria, cuya finalidad es ilustrar, celebrar, entretener o retar la experiencia de un grupo de personas".

Los eventos (culturales, artísticos o deportivos) crean una gran oportunidad para el desarrollo turístico de ciudades, regiones o países incentivando el desarrollo socioeconómico local, ayudando a la creación de empleo y a la creación y mejora de las infraestructuras (carreteras, hoteles, restaurantes, comercios, etc.), todo esto beneficia los turistas y la población local (Fernández, 2014). En esta línea, Henderson et al. (2010) afirman que "ciertos eventos tienen la capacidad de atraer a un gran número de turistas, por eso los agentes públicos buscan atraerlos por su contribución actual y potencial en el desarrollo del turismo y marketing”. Latiesa y Paniza (2006) afirman que existe una interconexión evidente entre turismo y deporte en las sociedades avanzadas y definen el turismo deportivo como "aquellas actividades relacionadas con el deporte que se realizan fuera del lugar habitual de residencia, no sólo por motivos competitivos, sino también por

Dirección para correspondencia [Correspodence address]: Patricio Sánchez Fernández. Universidad de Vigo (España).

E-mail: patricio@uvigo.es motivos recreacionales, ya sea en instalaciones artificiales o en el medio natural para realizar actividad física u observar espectáculos deportivos, pudiendo o no existir fines competitivos". Esta modalidad turística creció durante los últimos años así como la celebración de eventos deportivos organizados por las ciudades para atraer a los turistas deportivos (Fernández, 2014).

Goldman y Johns (2009) consideran el deporte como "un sector económico importante a nivel individual, organizacional y nacional y es importante su contribución en la actividad económica y en la creación de riqueza”. Bajo la óptica de Walker y Enz (2006), los deportes a menudo crean beneficios económicos indirectos. Estos autores afirman que la cuantificación del impacto económico de los deportes es difícil de medir y sugieren que el efecto del impacto económico se maximiza cuando los visitantes gastan dinero porque traen dinero "nuevo" a la economía local.

Un estudio sobre el impacto económico en un acontecimiento deportivo permitirá conocer si la inversión realizada va a proporcionar un beneficio tanto al territorio en el que se celebra como a sus habitantes. El impacto que puede generar puede ser tanto positivo como negativo, sobre todo en el caso de eventos deportivos de menor magnitud, ya que aunque no creen tanto interés sí pueden llegar a crear beneficios.

Se debe destacar la existencia de informes y estudios basados en el impacto económico de un acontecimiento deportivo. No obstante, en la modalidad de la natación son escasos. Esto puede ser debido a que la natación en comparación con 
otros deportes no se considera mayoritario por no ser de los más populares y seguidos por los espectadores tanto a nivel nacional como internacional y en directo por televisión. Entre los estudios de impacto económico de eventos de natación destacan los realizados por el organismo responsable del Deporte (Sport England) en 1999, Wislon (2006), Parent y Seguin (2007) y la Consultora Ipsos MORI North (2008).

El "posicionamiento del destino" supone un factor importante y es un término utilizado en marketing para conocer el lugar en orden de importancia que ocupan en la mente de los consumidores determinados productos. Algunos destinos consiguen alcanzar lugares destacados en dichas jerarquías que se consolidan como auténticas "mecas" en la práctica de determinados deportes (Latiesa y Paniza, 2006). Además, el éxito o fracaso en los eventos deportivos estará condicionado en su mayor parte por las estrategias y herramientas de comunicación utilizadas (Fernández, 2014).

Entre los cinco tipos de turismo deportivo (Resort, Cruises, Atracttions, Tours, Events) mencionados por Latiesa y Paniza (2006) se encuentran los de Events, cuyo objetivo es que los turistas sean espectadores de eventos deportivos.

Si el foco se centra en la celebración de eventos deportivos de reducida dimensión como es el caso del XXI Campeonato de Espańa “Open"” de Invierno Master de Natación celebrado en Pontevedra, este tipo de eventos pese a ser de menor escala, tal y como afirman Walo et al. (1996), pueden ser eventos especiales en relación a su importancia regional y local.

Cabe esperar que la comercialización de un evento traiga beneficios sociales, físicos y económicos para la comunidad local o la nación. Una característica común de cualquier evento es la anticipación de los turistas y el aumento de los gastos en el área. Existe la expectativa de que los gastos de los visitantes ayudarán de manera significativa a la economía local. Son muchas las comunidades locales que fomentan el deporte, la recreación y las oportunidades de turismo para hacer un completo uso de sus instalaciones recreativas y ampliar y diversificar su economía local. Si se hace una distinción entre los grandes acontecimientos y los eventos locales más pequeños se observa que existe una clara distinción entre ambos, ya que los de mayor escala provocan un efecto acelerador de la inversión de capital y altos costes de oportunidad potenciales, mientras que los otros generan un nivel marginal de la actividad turística que se puede satisfacer con los recursos existentes (Walo et al., 1996).

Los eventos especiales locales de una región debido a la euforia que los rodea pueden generar costes económicos importantes, esto supone incluir costes directos (servicio policial, recolección de basura, control del tráfico, etc.), indirectos (interrupción del estilo de vida, gamberrismo, etc.) y de oportunidad. Entre los beneficios que pueden crear se pueden incluir aumentos en el empleo, la inversión, los ingresos, servicios adicionales, infraestructuras y mejoras o crecimiento en el espíritu y la cooperación de la comunidad. Los eventos locales deben proporcionar un estímulo económico y tener un impacto social en la comunidad de acogida de una manera positiva (Walo et al., 1996).

A la hora de organizar un evento existen factores que condicionan la realización de un evento deportivo y otros que influyen en la generación de impacto económico. Autores como Magaz y Fanjul (2012), Ayora et al. (2004) y Gil (1995) citan ciertos elementos que condicionan el diseño y celebración de un evento deportivo diferenciándolos entre factores externos e internos.

Entre los factores externos, Magaz y Fanjul (2012) citan los factores políticos, el grado de interés para el municipio/ comunidad autónoma/País, el apoyo de diferentes instituciones públicas y privadas, factores demográficos, el entusiasmo y apoyo de los ciudadanos, el alcance mediático del evento, factores económicos, las infraestructuras y equipamientos, el factor impacto, el factor técnico, el tipo de cliente para el cual se va a organizar el evento y los stakeholders. Entre los factores internos estos autores destacan el factor organizacional, el factor experiencia, el factor tiempo, los recursos propios y el coste de oportunidad.

Por otro lado, Sánchez et al (2014) enumeran los factores que influyen en la generación de impacto económico. Consideran como significativos la modalidad (individual o colectivo), el tipo de competición, el ámbito geográfico, la duración del evento, los participantes, la federación, los espectadores, el espacio, las infraestructuras, la periodicidad de celebración y la tipología del evento. Todos estos elementos son importantes identificarlos pues ayudan a obtener una concepción más clara del impacto que engendran los eventos deportivos y determinar su celebración.

\section{Metodología}

El estudio realizado se centra en el análisis de los efectos económicos derivados de la celebración, en el municipio de Pontevedra, del XXI Campeonato de España "Open” de Invierno Master de Natación entre las fechas del 19 y 22 de febrero del 2015. El espacio territorial de referencia es ayuntamiento de Pontevedra. En este caso, no se tuvo en cuenta el gasto de los asistentes o miembros de la organización que residan en el municipio pontevedrés. Tampoco se contabilizaron los patrocinios de las empresas e instituciones pertenecientes el ayuntamiento de Pontevedra. Lo que realmente es relevante y de interés, es el análisis del gastos y patrocinios hechos por las personas o entidades no residentes en Pontevedra.

La exclusión de los residentes en Pontevedra movilizados por la celebración de este campeonato se justifica ya que, en el caso de que no se celebre el evento, realizarían un gasto semejante en actividades alternativas en el propio territorio pontevedrés y por lo tanto, no existe efecto añadido. Por el 
contrario, las rentas de los no residentes sí deben de computarse, ya que de que no se haya celebrado el campeonato, ese gasto se realizaría en otros territorios. De esta manera, la celebración del evento atrae la riqueza a la ciudad.

También se dejaron fuera del estudio algunos efectos (negativos y positivos) que van mas allá del estrictamente económico. En particular las molestias provocadas a los residentes en Pontevedra por la organización del evento, el incremento del bienestar en las personas que valoran positivamente la celebración de un campeonato de estas características, en concreto, en las personas que asistieron lo mismo y reducciones del bienestar en las personas que valoran negativamente la celebración del campeonato, particularmente, aquellas personas que se ven afectados por algún problema concreto derivado de su celebración.

Por último, cabe destacar que este estudio no mide la incidencia del evento sobre visitas futuras de turistas, porque eso requeriría hacer una encuesta continua en los meses próximos que permitiera conocer que porcentaje de las personas que acuden a Pontevedra como turistas, vienen influenciados por la celebración del campeonato.

\section{Enfoque metodológico: análisis costo-benefio (ACB)}

Walo et al. (1996) argumentan que existen dos grandes enfoques utilizados para la estimación de los impactos económicos para eventos especiales, los cuales son los modelos de ordenador (implican modelos de entrada-salida o de equilibrio adecentados para la modelización regional y nacional) y la contabilidad de gestión (implica estimar el gasto de los visitantes y el desarrollo de una comprensión de donde sucedieron los gastos). Un factor importante que consideran estos autores, a la hora de evaluar el impacto de este tipo de eventos, es el gasto de capital requerido para organizarlos. Los eventos que tienen costes de estatificación bajos y pocos o ningún gasto de capital tienen un mayor potencial de impactar positivamente en las economías regionales.

Sánchez et al. (2012) también exponen diferentes métodos empleados para medir el impacto económico entre los cuales mencionan seis: las Cuentas Satélite, las Tablas Input-Output, el modelo de Equilibrio General Computable (EGC), la Valoración Contingente, el Análisis Sectorial-Regional y el Análisis Coste-Beneficio (ACB).

Las Cuentas Satélite presentan un enfoque macroeconómico, con un ámbito de aplicación nacional o regional y que consisten en un conjunto de tablas estadísticas, basadas en la metodología de la Contabilidad Nacional, con capacidad de aportar parámetros e indicadores económicos del campo o actividad económica analizada (Lera, 2010).

Las Tablas Input-Output adoptan un enfoque macroeconómico de ámbito nacional o regional, posibilitando la elaboración de complejos modelos económicos de simulación del impacto bajo diversos escenarios (Pedroso y Salvador, 2003).

Los modelos de Equilibrio General Computable (EGC) consisten en una representación realista de la economía, realizada mediante un grupo de ecuaciones que representan la producción, el consumo y el comercio del sector privado, así como las actividades económicas del sector público. Sin embargo, como seńalan Rodríguez et al. (2011), los efectos de mercado recogidos por los modelos EGC no son significativos para eventos de pequeño tamaño el cual limita su aplicación práctica.

El Método de Valoración Contingente consiste en observar la reacción de un individuo ante cambios hipotéticos (contingentes) en los precios o cantidades de bienes o servicios (Leal, 2005).

Finalmente, el análisis sectorial permite obtener datos precisos y detallados sobre las actividades socioeconómicas vinculadas al deporte o el máximo de informaciones posibles sobre los componentes de la oferta y demanda de bienes y servicios deportivos y de las actividades socioeconómicas, para visualizar la influencia económica del deporte en el área geográfica objeto de estudio.

Las metodologías existentes no son excluyentes entre sí, por el contrario se complementan. Métodos como las Cuentas Satélite o las Tablas Input-Output, aportan datos útiles para la realización de cálculos a partir de un Análisis Coste-Beneficio o un Análisis Sectorial-Regional. Por su banda, como seńalan Barajas et al. (2012), la Valoración Contingente aporta medidas de beneficio y coste de los consumidores o datos como el valor de no uso, que enriquecen y amplían el alcance de los otros métodos.

En este estudio, el método empleado fue el ACB. Este método resulta muy eficaz para evaluar los impactos socioeconómicos derivados de la celebración de los eventos. Es una herramienta importante para la toma de decisiones por parte de los responsables de la organización de los mismos. Este método puede llevarse a cabo, ex-ante (con la utilización de estimaciones), o bien ex-post (a partir de los datos reales registrados) si queremos evaluar el éxito o fracaso (Ramírez et al., 2007) o de los datos que desee encontrar.

La utilización de este método es habitual en los estudios sobre transportes (Rise y Macian, 1998) o en sanidad (Póveda et al., 2004; Velasco y Rodríguez-Monguió, 2009). En relación al análisis de costes y beneficios relacionados con el deporte, esta metodología fue recomendada por Burns et al. (1986) para la medición del impacto económico del Gran Premio de Fórmula 1 en Adelaida. Recientemente, el método fue utilizado por Ramírez et al. (2007) en su estudio sobre el impacto económico y social de la celebración de grandes eventos a nivel local.

La aplicación de este método debería realizar siguiendo una serie de fases. En la primera fase, se identificarán los cos- 
tes y beneficios que el proyecto supone para las sociedades, y estos se clasificarán según sean puntuales o recurrentes. Para la valoración de estos costes y beneficios repetitivos se utiliza el Valor Actual Neto (VAN). Por otro lado, también se hace distinción según sean tangibles o intangibles. En la segunda fase, los costes y beneficios deben ser cuantificados en unidades monetarias para que sean fácilmente comparables, y se pueda obtener un resultado interpretable económicamente. En la tercera y última fase, se procede al cálculo de dicho resultado restando los costes a los beneficios. Si el resultado es positivo, el proyecto será deseable, mientras que en el caso de obtener un resultado negativo, se trataría de una actividad poco adecentada desde la dimensión económica. En el caso de una actividad recurrente, quiere decir que a diferencia de beneficios menos costes, irán produciéndose a lo largo del tiempo. Para obtener el valor presente, se actualizan los flujos derivados del evento, obteniendo su Valor Actual Neto (VAN) si en el primer período deducimos el desembolso realizado para la celebración del evento. Para la actualización de la corriente de beneficios y costes se emplea una tasa de descuento, que refleja la mínima rentabilidad exigida. Si el resultado de esta actualización es positivo, el proyecto será deseable. Otra alternativa para evaluar si el proyecto o actividad es financieramente viable, consiste en calcular la relación entre los beneficios y los costes. En este caso, si el valor del prorrateo de beneficios entre costes es mayor a la unidad, la alternativa es ventajosa, ya que nos indica que proporciona una rentabilidad al ser mayores los beneficios que los costes.

El método ACB presenta importantes ventajas como son su utilidad en la toma de decisiones sobre proyectos a elegir o realizar, el uso de variables tangibles e intangibles influyentes en el impacto económico de las actividades llevadas a cabo y el cálculo de la rentabilidad que proporciona el proyecto. Además, tiene en cuenta el coste de oportunidad de llevar a cabo cualquier otro proyecto con los mismos recursos utilizados (Ramírez et al., 2007). No obstante, cuenta con una serie de limitaciones a la hora de aplicarlo. La más importante es la necesidad de una abundante cantidad de información, la dificultad para valorar monetariamente las variables intangibles y el problema para elegir una tasa de descuento.

La realización del análisis ex-ante supone la utilización de estimaciones para predecir la corriente de beneficios y costes que producirá el evento. La evaluación ex-post del evento se realiza a partir de los datos reales registrados (Ramírez et al., 2007). En este caso se realiza una evaluación ex-post.

Los efectos tangibles pueden ser beneficios o costes para el ámbito donde se realiza el evento. Los positivos se analizan en varias categorías relativas a los gastos de las personas involucradas en la organización del evento y principalmente, los asistentes que acuden al lugar de celebración. Por otro lado, los ingresos recibidos de los patrocinadores no situados en el municipio también producen un beneficio tangible para
Pontevedra. Por su parte, el único coste que se computa va a ser el derivado de los gastos realizados por la organización del evento fuera de la ciudad. La suma de los efectos, positivos y negativos, proporciona el valor del impacto económico consecuencia del evento objeto de estudio: XVII Campeonato de España "Open” de Invierno Master de Natación. En este caso, el análisis se simplifica porque se estiman los efectos en un ańo base, sin tener que realizar actualizaciones de valores. Por otro lado y como ya se indicaba en la introducción, los efectos intangibles se descartan por motivos de limitaciones del estudio.

\section{Técnicas de obtención de datos}

La información necesaria para la realización del estudio sobre el impacto económico de la organización del Campeonato Master de Espańa en Pontevedra en el mes de febrero de 2015 se consiguió mediante la realización de entrevistas a los organizadores y una encuesta a los asistentes, ya fuera como participantes o como espectadores. Este procedimiento es lo utilizado por autores como Ramírez Hurtado et al. (2007).

Las entrevistas a la organización fueron tanto previas como con posterioridad al evento. Por otro lado, desde la organización se facilitaron los datos solicitados y respondieron a las cuestiones que se le realizaron. Los participantes y espectadores fueron encuestados por un equipo de encuestadores de la propia Universidad de Vigo, en los días del evento, en la piscina donde se realizó la competición.

En total, se encuestaron 324 personas lo que implica una margen de error del 5\% con un nivel de confianza del 95\%. Para garantizar la aleatoriedad de la encuesta, los encuestadores siguieron un patrón de conducta dirigiéndose a una persona de cada tres en cada zona y realizándolas en diversos momentos a lo largo de las jornadas. El texto de la encuesta a los espectadores se estructura en cuatro partes diferenciadas con un total de 26 preguntas, que se describen a continuación.

La primera parte del cuestionario aborda la identificación en términos deportivos de la persona encuestada, entre las que destaca su condición de participante en la prueba o sólo espectador y, en caso de que participe, el club de pertenencia. Además, en esta primera fase también se recoge si la persona encuestada y residente en Pontevedra o no, en el caso de que la respuesta sea negativa, se recogerá el lugar de procedencia.

La segunda parte recoge preguntas referentes a la planificación del evento por parte de los asistentes, como el número de días que pasaron en Pontevedra, como se percataron de la celebración, si acude a la ciudad con motivo del evento, dónde pasaron la noche y cuantos días, si acudieron al evento en años anteriores o qué actividades realizaron. En este apartado también se incluyen los aspectos económicos relacionados con los asistentes. Concretamente se pregunta sobre la cantidad de dinero que tenía intención de gastar en la ciudad y su 
repartición. Dado que la experiencia dice que una pregunta tan directa resulta difícil que sea contestada, se optó por proponer tres tipos de preguntas diferenciadas para poder tratar los datos de la manera más rigurosa posible. Se preguntaban por orden y, si no se obtenía respuesta, se ofrecía la siguiente como alternativa.

La primera de ellas analiza el gasto efectuado en Pontevedra por conceptos. Si el encuestado no es capaz (o no quiere) distribuir el gasto, se le ofrece la opción de indicar el montante global del gasto, y si aún así tampoco responde, se facilita un rango de intervalos entre los cuáles estima que se situaría su gasto.

La tercera parte se enfoca en conocer la vertiente turística de los encuestados. Se incluye puesto que, aunque no es cuantificable, sirve de orientación sobre su posible incidencia futura en el turismo con su consecuente rendimiento económico. Así, se preguntaba si vinieron en años anteriores, qué zona de la ciudad le gusta más y cuál menos, si volvería en años posteriores, la puntuación sobre la imagen de la ciudad para el evento y la comparación de los mismos con otros similares a los que habían asistido, si está satisfecho con la organización del evento, si es seguidor de eventos deportivos o cuáles son las preferencias deportivas.

La cuarta parte recoge aspectos socio-demográficos de las personas encuestadas tales como edad, sexo, profesión, nivel educativo o el tramo de ingresos individuales anuales. También resulta de interés en este apartado preguntar por el número de acompańantes.

Finalmente, una vez recogida toda la información procedente de las encuestas se procesó para el posterior análisis de los datos.

\section{Estimación del impacto económico}

Estimaciones relativas al gasto

En la encuesta se preguntó sobre los gastos realizados en la ciudad. La experiencia indica que las preguntas que más reticencias provocan a la hora de responder las encuestas son los referentes a aspectos económicos. Por ese motivo, se formularon tres posibilidades a la hora de responder: analizar el gasto, dar un valor estimado del gasto en conjunto o señalar tramos de gasto. Se preguntan por esta orden pues es la forma de lograr datos más precisos. Resulta relevante indicar que se obtuvo un grado de respuesta sobre los detalles económicos del $98,4 \%$.

Un total de 284 personas contestaron analizando por conceptos el gasto que estimaban que iban a realizar. El importe medio de gasto que habían previsto realizar resulta de 279,7 euros. Otras 7 personas contestaron con la cifra global estimada de su gasto en la ciudad. En este caso, el importe medio resultante es de 267,9 euros.
Para determinar el gasto del grupo de personas que contestó dando importes por tramos, se utilizan las marcas de clase. Dado que el tramo superior no se indicaba límite, se tuvo que determinar un valor para ese intervalo. Se optó por tomar como referencia la información de los asistentes que, especificando sus gastos, dijeron que globalmente gastaron más de 600 euros (límite del tramo anterior). Así, hay un total de 10 personas que estimaban un gasto superior a ese importe. Haciendo la media de los valores de esas personas resultan que el gasto sería de 736 euros. Este importe es el que tomamos como marca de clase para el último tramo.

Una vez calculadas las marcas de clase, se determina el número de personas que contestó en ese intervalo (frecuencia). Posteriormente se multiplica la marca de clase por la frecuencia. La Tabla 1 muestra los resultados del cálculo del gasto por intervalos.

Tabla 1. Cálculo de gastos para las respuestas por intervalos.

\begin{tabular}{lccc}
\hline Intervalo de gasto $(€)$ & $\begin{array}{c}\text { Marca de clase } \\
(\mathrm{xi})\end{array}$ & $\begin{array}{c}\text { Frecuencia } \\
\text { (fi) }\end{array}$ & ${ }^{\text {xi*fi }}$ \\
\hline $0-100$ & $50,0 €$ & 5 & $250,0 €$ \\
$100-300$ & $200,0 €$ & 3 & $600,0 €$ \\
$300-600$ & $450,0 €$ & 3 & $1.350,0 €$ \\
$600-940$ & $736,0 €$ & 1 & $736,0 €$ \\
\hline TOTAL & & 12 & $2.936,0 €$ \\
\hline
\end{tabular}

Fuente. Elaboración propia a partir de las encuestas.

Para el cálculo del gasto medio por persona, se realiza una media ponderada del gasto obtenido en función del conjunto de respuestas. Como ya se mencionó, gracias a ofrecerse diversas alternativas para contestar, se obtuvo un grado conjunto de respuesta del $98,4 \%$, el que garantiza la significatividad estadística al tiempo que se puede considerar como un porcentaje de respuesta altamente satisfactoria para un estudio como el aquí llevado a cabo.

La Tabla 2 muestra la estimación de gasto medio por persona asistente al evento. Se optó por trabajar con la media ponderada que resultó en 278 euros de gasto por persona.

Tabla 2. Cálculo de la media ponderada de gasto por persona asistente al evento.

\begin{tabular}{lccc}
\hline Pregunta & Importe del gasto & Personas & Media de gasto por persona \\
\hline 12 & $79.425,5 €$ & 284 & $279,7 €$ \\
12.1 & $1.875,0 €$ & 7 & $267,9 €$ \\
12.2 & $2.936,0 €$ & 12 & $244,6 €$ \\
\hline \multicolumn{4}{r}{ Media ponderada } \\
\hline
\end{tabular}

Fuente. Elaboración propia a partir de las encuestas

1 La marca de clase para cada intervalo de gasto (xi) representa el punto medio de cada uno, al tiempo que frecuencia (fi) determina el número de veces que aparece cada valor.

Impacto económico del XXI Campeonato de España “Open” de Invierno Master de... SPORT TK, 5 (supl.), 169-180 
Estimaciones relativas a los asistentes

Una competición como la estudiado presenta algunas ventajas a la hora de estimar al número de asistentes. En primer lugar, el hecho de que los participantes estén inscritos. En segundo lugar, la celebración en un recinto cerrado. Y, finalmente, que en gran medida, los participantes en la prueba son a su vez espectadores. Así, en este caso, se partió del número de inscritos en la prueba para realizar los cálculos.

No fue posible obtener el número de no presentados y bajas del campeonato, por lo que se asumió un porcentaje muy bajo de tal forma que fuese despreciable a efectos de nuestras estimaciones. Esta asunción está fundamentada en el hecho de que, como se recoge en la normativa de la competición, en los casos de la no presentación a una prueba, sin ser comunicada previamente por el club, se aplicarán las sanciones previstas por este concepto tanto en el Reglamento General de la RFEN 2 como en la Normativa de Aspectos Generales ${ }^{3}$.

Por ese motivo, se empleó el dato de 1.272 inscritos para realizar los cálculos del impacto económico. A esa cifra le restamos los inscritos de los dos clubes de Pontevedra, C.N. Galaico y C. N. Master Pons Vetus, con 13 y 10 participantes, respectivamente. Así, el dato con el que se trabajó fue de 1.249 personas.

Además de los participantes, asistieron algunos espectadores que no competían. En la encuesta, se preguntaba a las personas si eran espectadores o participantes. El resultado fue que el 13,6 \% de los encuestados eran espectadores. A partir de ese dato se obtuvo, que cada 6,3 participantes había un acompańante o espectador.

Eso implica que, sobre 1.249 participantes, habrían venido al evento 198 espectadores. Eso supone que el total de personas con impacto económico en la ciudad sería de 1.447 . Aunque la cifra de espectadores podría parecer baja no lo es, dadas las características del evento analizado en la que muchos acompañantes también son nadadores.

A esta cifra hay que descontarle aquellos que pensaban venir en otro momento y cambiaron la fecha haciéndola coincidir con el campeonato (time-switchers) y aquellos que participan en la competición porque se encontraban ya en la ciudad (casuals). En este caso, el porcentaje de time-switchers es muy bajo, sólo el 0,31\%, y no hay casuals. Esto supone que habría 4 personas que serían time-switchers.

En la Tabla 3, se presenta la estimación del número de personas que generan impacto económico en la ciudad de Pontevedra con motivo del Campeonato Master de Natación.

2 Es decir, no pueden participar en ninguna otra prueba durante esta jornada

3 http://www.rfen.es/publicacion/campeonatos/files/ rfen100/2015/2015XX100004/normativa.pdf
Tabla 3. Número de personas que generan impacto económico.

\begin{tabular}{cccc}
\hline No residentes & Time-switchers & Casuals & Personas con impacto \\
\hline 1.447 & 4 & 0 & 1.443
\end{tabular}

Fuente. Elaboración propia a partir de las encuestas

Estimación del impacto de participantes y espectadores

Una vez determinadas las personas que van a ocasionar una repercusión económica en la ciudad, se multiplica esa cifra por el gasto medio ponderado por persona según lo estimado en la tabla (tabla de la media ponderada). El resultado es el que aparece en la Tabla 4.

Tabla 4. Impacto económico de los asistentes

\begin{tabular}{ccc}
\hline $\begin{array}{c}\text { Personas con } \\
\text { impacto }\end{array}$ & $\begin{array}{c}\text { Gasto medio por } \\
\text { persona }\end{array}$ & Impacto económico \\
\hline 1.443 & $278,0 €$ & $401.154 €$ \\
\hline
\end{tabular}

Fuente. Elaboración propia a partir de las encuestas

Por lo tanto, el importe que los asistentes al Campeonato Master de Natación gastaron fue de 401.154 euros.

Estimaciones relativas a la organización

Para efectuar la estimación directa del impacto económico se preguntó a la Organización sobre los gastos en qué incurrieron para celebrar el Campeonato, y qué ingresos obtuvieron para financiarlo. La Organización proporcionó los datos económicos relativos al Campeonato de Espańa "Open” de Invierno Master, desglosados en aquellos que fueron realizados fuera de la ciudad de Pontevedra y los que se efectuaron en ella.

A partir de estos datos se determinaron los efectos negativos y positivos que tuvo la celebración del campeonato para la ciudad.

\section{Resultados}

\section{Perfil de los participantes-espectadores}

Otro de los objetivos del estudio es conocer el perfil de los asistentes al Campeonato de España "Open" de invierno Master, incluyendo tanto a los participantes como los espectadores. Para ello, en la encuesta se formularon una serie de preguntas que ayudan a definir el público asistente.

El primer asunto de interés y que, de hecho constituye una característica muy relevante de esta competición es, que una amplia mayoría de los asistentes a este evento son participantes (86,4\%). Esto es, sólo un 13,6\% de los encuestados son espectadores. 
Por lo que se refiere a las características demográficas habituales en este tipo de estudios hay que señalar que un $64,3 \%$ son hombres y un $35,7 \%$ de los asistentes son mujeres. Esta pregunta además sirve como control sobre la calidad de la muestra. Dado que en la inscripción también se especifica el sexo, se verifica el porcentaje sobre los datos de inscripción y se compara con lo obtenido de la encuesta. La variación es inferior al porcentaje del 5\% que considera el error muestral. Esto supone una garantía adicional sobre la bondad de la muestra.

Al tratarse de un Campeonato de ámbito estatal, los asistentes a este evento proceden de casi todas las comunidades de España. No obstante, se da una importante concentración de personas procedentes de determinadas regiones, a saber: Madrid (20,5\%), Barcelona (9,4\%), Valencia (5,2\%), Asturias $(4,2 \%)$ y Cantabria $(3,9 \%)$. Este hecho viene motivado porque la mayoría de los equipos participantes provienen de estas zonas que, además, son sedes de los equipos más importantes a nivel nacional en la natación master. Si se distribuye a los asistentes al campeonato en función del área geográfica de España, observamos como a un $43,6 \%$ procede del noroeste del país, mientras que $24 \%$ procede del nordeste.

Atendiendo al nivel educativo de los entrevistados, se observa como un 79,2\% manifiesta que tiene un título de educación superior, mientras que el 10,7\% solo alcanza el nivel educativo de formación profesional. En el sondeo realizado, no se encontraron asistentes sin estudios.

Asimismo, referente a la profesión tampoco se observa un perfil claramente definido. La ocupación de los asistentes es muy dispar. En todo caso cabe señalar que las profesiones que más se repitieron fueron: jubilado (8\%), estudiante, ingeniero y maestro (7\%), monitor de natación (5\%), funcionario $(5 \%)$ y administrativo (4\%).

En esta encuesta también aparecen evaluados el nivel de ingresos de los asistentes. Un 28,7\% manifiesta que sus ingresos superan los 30.000 euros anuales. Por otra parte, el $22,8 \%$ declara que su nivel adquisitivo se encuentra entre los 20.000 y 30.000 euros.

Otro aspecto relevante de los asistentes al campeonato viene dado por su edad. Por la propia configuración del evento, este aspecto resulta de un perfil muy variado. En concreto, la edad media de los asistentes ronda los 41 ańos, no obstante, el porcentaje de asistentes menores de 41 años es del 53\% y el porcentaje de asistentes con una edad igual o superior a la media es del $47 \%$. Así pues, puede afirmarse que sobre la edad no hay un perfil claro definido, esto se debe a que hay asistentes jóvenes, de mediana edad y de edad más avanzada.

Con respecto a las cuestiones organizativas del evento, un aspecto de interés se refiere a cómo se percataron de la celebración del Campeonato de España “Open” de Invierno Master. En este caso, lógicamente, más del $80 \%$ de las personas encuestadas reconoce que se percató a través del club al que pertenece. Por otro lado, un $10 \%$ de los encuestados confiesa que se percató del evento ya que conoce a participantes, mientras que solo un $8 \%$ manifiesta que se percató a través de los medios de comunicación.

Igualmente, de la encuesta se desprende que casi el 100\% de los asistentes al evento acudieron únicamente con motivo de la celebración del campeonato. Sólo un 0,32\% asistieron por otros motivos, por lo que se consideran los denominados casuals o time-switchers.

Los asistentes aprovechan esta visita para realizar otro tipo de actividades en la ciudad. Un 88,3\% de los encuestados afirma que comería en restaurantes de la ciudad, y un 37,0\% también manifestó su intención de realizar compras en esta.

En las encuestas también se observa que un 66,9\% de los asistentes acudió a este campeonato en ediciones anteriores, un $33,1 \%$ reconoce no haber asistido al campeonato en otras ocasiones.

En relación a la asistencia a este evento hay que tener en cuenta que el campeonato tenía una duración de 4 días (de jueves a domingo). Por lo tanto, la media de los días que los asistentes pasaron en la ciudad $(3,8)$, casi coincide con esta cifra. A partir de esto cabe mencionar que un $88,0 \%$ reconoce que pas la noche en la ciudad y alrededores y sólo un 12,0\% no el fijo. La gran mayoría (un 55,5\%) manifiesta que pasará 3 noches en la ciudad. El alojamiento escogido por la gran mayoría y el que prefieren los encuestados es el hotel. Dicha opción representa un $81,5 \%$.

Si se hace referencia al gasto realizado por los asistentes en la ciudad durante su estancia, cabe destacar que principalmente realizaron gasto en alojamiento, donde el gasto medio fue de $119,6 €$ por persona (un 32,8\% del gasto total que van a realizar). Manutención es el siguiente en el que más gastaron los encuestados, la media de gasto en comida fue de $93,6 €$ por persona (25,7\% de su presupuesto). En tercer lugar están las compras, puesto que los asistentes se gastaron de media unos $64,4 €(17,7 \%)$. En turismo la media de gasto es de $29,4 €(8,1 \%)$, en desplazamiento el gasto medio es de $22,6 €$, y finalmente la media gasto en ocio es de $6,8 €(1,9 \%)$.

La totalidad de los encuestados afirma que le gustaría volver en años posteriores a Pontevedra. Más en concreto, esta afirmación la realizó un $97,5 \%$ de los encuestados. Mientras que, un 1,3\%, manifiesta que quizás volvería, y el otro 1,3\% restante nunca lo haría.

Mayoritariamente, un 97\%, confirmó sentirse satisfecho con la organización del Campeonato de Espańa "Open" de invierno Master.

Tanto la imagen de la ciudad de Pontevedra como lugar para celebrar el evento y la imagen del evento en general, recibió una puntuación muy positiva por parte de los encuestados, en ambos casos es superior al 4, siendo la puntuación máxima uno 5. En cuanto a la imagen de Pontevedra como un lugar de destino de vacaciones la puntuación obtenida 
también fue positiva, obteniendo un 3,8 de media.

Con respecto a la pregunta de si los encuestados son seguidores de eventos deportivos, un 95,8\% manifiesta que sí, mientras que sólo un 4,2\% declara que no es seguidor.

A la hora de comparar este evento con otros campeonatos de este estilo a los que acudieron, no existe una tendencia clara. Un $42,2 \%$ piensa que este evento es mejor, mientras que el otro $42,2 \%$ considera que el campeonato de Pontevedra es igual a los demás campeonatos de su categoría. Un 7,8\% lo consideraron mucho mejor y sólo un $6,2 \%$ lo consideró peor que otros campeonatos del estilo que ya conocían.

Por último, para terminar de definir el perfil del espectador, se les preguntó por sus preferencias deportivas. Evidentemente un $41,5 \%$ reconoce ser aficionado a la natación. Pero cabe destacar que un $13,2 \%$ es aficionado al fútbol, casi un $8 \%$ reconoce que le gusta el tenis, un $7,4 \%$ es aficionado al ciclismo y un $7 \%$ al baloncesto. Estas son las aficiones deportivas que más destacaron entre los asistentes al campeonato sin contar la natación.

\section{Información turística}

En la encuesta también se les preguntó a los asistentes acerca de cuestiones relacionadas con el turismo y la imagen que ellos tienen de la ciudad de Pontevedra.

De las personas que contestaron acerca de su zona favorita de la ciudad de Pontevedra, puede concluirse la preferencia de una amplia mayoría por el casco viejo o la zona antigua de la ciudad (53,1\%). A considerable distancia, lo sigue en preferencias la parte de la Ría $(21,7 \%)$.

En lo referente a las zonas que menos les gustaron de la ciudad, las más mencionadas por los encuestados fueron, la planta de celulosa $(29,4 \%)$, la zona que rodea la piscina en la que se celebró el campeonato $(23,5 \%)$ y los alrededores de la ciudad de Pontevedra (17,6\%).

\section{Impacto económico positivo para la ciudad de Pontevedra}

Desde el punto de vista económico, son positivos para la ciudad aquellos gastos que entidades de fuera de Pontevedra hicieran en ella, los ingresos obtenidos para financiar el evento y las inversiones o subvenciones percibidas de patrocinadores o entes públicos de fuera de la ciudad. No obstante, en esta edición del campeonato, la organización no contó con ingresos para su celebración según los datos facilitados por el club organizador.

Esta situación está pendiente de una negociación específica entre el club y el ayuntamiento de cara a compensar los gastos incurridos. No obstante, al proveer de las arcas del Ayuntamiento no se altera la estimación del impacto económico.

\section{Impacto económico negativo para la ciudad de Pontevedra}

El impacto económico negativo proviene de los gastos que la organización realiza fuera de la ciudad de Pontevedra. Es decir, aquellos fondos de la ciudad o conseguidos por la organización que se trasladaron a otros lugares. Para la celebración del campeonato, la organización tuvo que acudir a proveedores de otros lugares de Galicia y de fuera de la comunidad gallega.

En la Tabla 5, se detallan los gastos ligados a la celebración del Master. Estos gastos aparecen analizados según tengan su origen en proveedores de la ciudad, proveedores del resto de Galicia y de otros lugares de España. Los que no se destinan a pagar proveedores de Pontevedra tendrán un efecto negativo.

Tabla 5. Desagregación de gastos según el origen de los proveedores.

\begin{tabular}{ll}
\hline Concepto & Importe \\
\hline $\begin{array}{l}\text { Gastos de la organización con provedores de la } \\
\text { ciudad }\end{array}$ & $18.884,4 €$ \\
$\begin{array}{l}\text { Gastos da organización con provedores del resto } \\
\text { de Galicia }\end{array}$ & $7.234,6 €$ \\
$\begin{array}{l}\text { Gastos da organización con provedores de fuera de } \\
\text { Galicia }\end{array}$ & $16.778,0 €$ \\
\hline
\end{tabular}

Fuente. Elaboración propia a partir de los datos de la organización

La Ilustración 1 presenta la repartición de los gastos que la organización realizó con proveedores procedentes de fuera de la ciudad de la Pontevedra. Esos gastos supondrán una reducción de recursos para la ciudad.

Ilustración 1. Gastos de la organización con proveedores de fuera de Pontevedra.

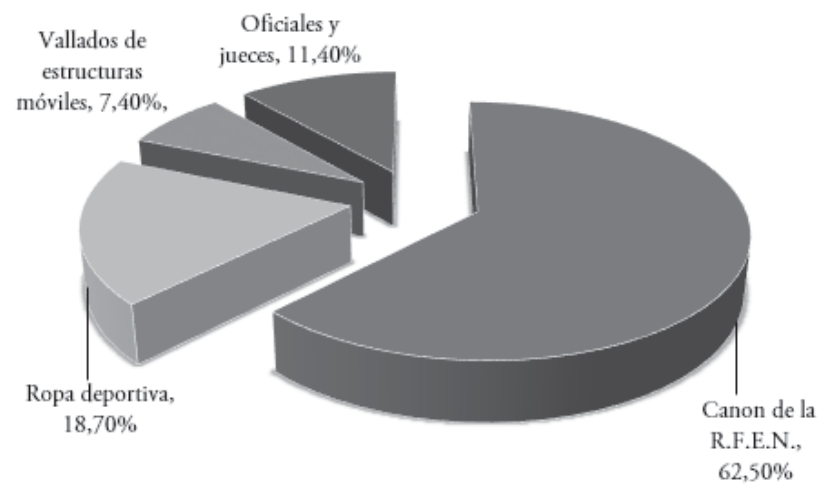

Fuente. Elaboración propia a partir de los datos de la organización 
Tabla 6. Gastos totales con provedores de fuera de la ciudad.

\begin{tabular}{ll}
\hline Concepto & Importe \\
\hline Gastos de la organización con provedores del resto & $7.234,6 €$ \\
de Galicia & \\
Gastos de la organización con provedores de fuera & $16.778,0 €$ \\
de Galicia & \\
Gastos totales con provedores de fuera de Ponte- & $24.012,6 €$ \\
vedra & \\
\hline Fuente. Elaboración propia a partir de los gastos de la organización
\end{tabular}

Como se puede observar en la Tabla 6, el importe de los gastos que realizó la organización, y que si fueron a otros lugares fuera de Pontevedra, es de 24.012,6 €. Este importe constituye el impacto económico negativo del evento.

\section{Impacto económico neto para la ciudad de Pontevedra}

A continuación, en la Tabla 7 se muestra, a modo de resumen, los gastos e ingresos que tienen repercusión en la ciudad de Pontevedra y sus importes totales.

Tabla 7. Gastos e ingresos de la organización con repercusión económica en la ciudad de Pontevedra

\begin{tabular}{ll}
\hline Concepto & Importe \\
\hline $\begin{array}{l}\text { Ingresos totales con repercusión en la ciudad de } \\
\text { Pontevedra }\end{array}$ & $0,0 €^{1}$
\end{tabular}

$(-)$

Gastos de la organización con provedores de fuera de Pontevedra

$24.012,6 €$

$(=)$

Recursos netos que salieron de la ciudad de Pontevedra $-24.012,6 €$

Fuente. Elaboración propia a partir de los datos de la organización.

Como conclusión puede decirse que los gastos en los que incurrió la organización fuera de la ciudad de Pontevedra fueron mayores que los ingresos percibidos por esta misma razón. Por lo tanto, suponen una reducción neta de recursos de 24.012,6 $€$ euros.

Una vez realizadas las estimaciones sobre el importe del impacto económico que generaron los asistentes y la organización es el momento de determinar el importe neto.

Tabla 8. Impacto económico neto del evento

\begin{tabular}{ll}
\hline Concepto & Importe \\
\hline Impacto económico generado por los asistentes & $401.154,0 €$ \\
- Recursos netos que salieron de la ciudad de Pon- & $24.012,6 €$ \\
tevedra & $377.141,4 €$ \\
= Impacto económico neto &
\end{tabular}

SPORT TK: Revista Euroamericana de Ciencias del Deporte
Tal y como se aprecia en la Tabla 8 , el impacto económico neto que supuso la organización del Campeonato Master de Natación en la ciudad de Pontevedra supera los 377.000 euros.

Habría que puntualizar que la cifra anterior debería corregirse teniendo en cuenta niveles de ocupación hotelera y de consumo en fechas similares a otros ańos. Esto es debido a que se podría argumentar que parte del gasto realizado por los asistentes al evento podrían haberla realizado turistas u otras personas que quizás dejaron de venir precisamente a consecuencia del evento. No obstante, se considera que la repercusión económica es tan elevada que aún con una importante deducción seguiría resultando un importante impacto económico positivo.

El hecho de no tener que realizar inversiones, aprovechando las infraestructuras existentes, contribuye a que la organización de este tipo de eventos consiga cifras tan beneficiosas.

Igual que se hace en estudios de este tipo, otra información de interés sería el impacto por día de celebración. Así, en este caso, dado que el evento se prolongó durante cuatro jornadas, el impacto diario resultante es de 94.285,35 euros.

Tabla 9. Resumen de la información del impacto económico

\begin{tabular}{ll}
\hline Concepto & Importe \\
\hline Impacto económico neto & $377.141,4 €$ \\
Impacto económico diario & $94.285,35 €$ \\
\hline
\end{tabular}

Fuente. Elaboración propia a partir de los datos de la organización

\section{Conclusiones}

Tras la realización del estudio sobre el impacto económico generado por la organización en Pontevedra del XXI Campeonato de Espańa "Open” de Invierno Master de Natación, el primer aspecto que debe ser concluido es que se trata de un evento claramente rentable para el ayuntamiento desde el punto de vista económico. Esta afirmación se encuentra refrendada por el hecho de que el impacto económico neto del campeonato se acerca a los 380 mil euros (377.141 €). Esto supone que por cada jornada del evento, el impacto económico fue de casi 100 mil euros $(94.285 €$ ) por cada uno de los cuatro días de celebración.

Procede aclarar la particularidad del hecho de no contar hasta el momento con ningún tipo de ingreso por parte de la organización. Desde el punto de vista del impacto económico este hecho no tiene repercusión. De esta manera el resultado señalado no se ve alterado. Además hay que incidir en el hecho de no tener que realizar inversiones específicas para la celebración del evento. Así pues, resulta crucial aprovechar las infraestructuras ya existentes y no incurrir en construcciones de instalaciones.

Otro punto que se manifiesta de vital importancia es hacer 
una selección certera tanto de la modalidad deportiva a celebrar como de las categorías objeto de competición. A partir de aquí, entran en juego otros muchos factores que van desde la tradición de ese deporte en el ayuntamiento como condicionantes políticos, educativos o sociales.

Sobre las categorías de los participantes, con carácter general y al margen de los grandes eventos deportivos (los mega-eventos), puede afirmarse que son dos las categorías que generan un mayor impacto económico en la zona. En primer lugar estarían las categorías más pequeñas, puesto que suelen mover a un gran número de personas que acompañan a los más pequeños de las familias. En sentido contrario, y cada vez con mayor importancia, la práctica deportiva y competitiva de los más mayores (veteranos, masters, de edades, etc. según el deporte que se trate) se presentan como eventos con clara repercusión económica allí donde se celebren al conjugar un doble perfil de deportista más turista.

Al hablar de turistas (turistas deportivos en este caso) conviene no olvidar los condicionantes turísticos del lugar de celebración así como sus limitaciones. En concreto, sería un error intentar organizar un evento que citara a más participantes que aquellos que la zona fuera capaz de albergar. Igualmente, otras circunstancias tales como la previsible celebración de otro evento, tanto en la zona como en el entorno próximo que limitara también la capacidad hotelera de la zona sería un handicap que habría que tener presente. Quiere esto decir que el planteamiento debería ser justamente el contrario y, en la medida de lo posible, utilizar el evento para desestacionalizar la demanda, con el cual ya se está apuntando aquí otro criterio para la selección del evento que es la no coincidencia con las épocas de temporada turística alta de la zona.

Otro aspecto desde el punto de vista turístico es el porcentaje de personas que acudió a Pontevedra exclusivamente por la celebración del campeonato. Dicho de otra manera, si no se hubiera celebrado el evento en Pontevedra esas personas acudirían a la ciudad (con casi toda seguridad estarían allí donde se celebrara) y, por lo tanto, no tendrían la oportunidad de visitar tanto la ciudad como su entorno. Los resultados del estudio reflejan como dos terceras partes de los asistentes no habían visitado Pontevedra (un 63\%) y otro 20\% solo había venido una vez.

Este es un rasgo fundamental del perfil del turista deportivo que se junta con otro muy relevante desde la perspectiva del organizador como es el elevado grado de satisfacción. La satisfacción del turista es la llave para conseguir su fidelización y en el caso de los turistas deportivos, dado que sus objetivos y motivaciones están más claros que los del turista "tradicional", resulta más fácil poder cumplir sus expectativas. En el caso del Campeonato de España Master de Natación, el estudio realizado refuerza esta circunstancia puesto que casi la totalidad de los asistentes son al mismo tiempo participantes en la prueba (prácticamente nueve de cada diez, un 86\%).
Quiere esto decir que para que la experiencia de los asistentes/ participantes sea favorable, incluso en términos turísticos, el evento debe ser correctamente organizado para queden satisfechos y puedan disfrutar y aprovechar del resto del tiempo (el que no dedican a competir). Consecuentemente, el grado de satisfacción con la organización fue muy elevado. Así, casi la totalidad de los encuestados (el 98\%) mostraron su satisfacción y la intención de volver a la ciudad.

Otra característica que debe ser reseñada es la capacidad económica de los participantes, puesto que facilita, sin duda, la obtención de un favorable impacto económico por la vía del gasto realizado en los días de la competición. Pese a que el deporte de la natación no se considera como un deporte caro o costoso para su práctica, casi un tercio de los asistentes (un 29\%) indican unos ingresos anuales superiores a los 30.000 euros.

Este nivel económico tiene su reflejo en el gasto realizado, de manera tal que la hostelería y la restauración fueron los sectores que más se beneficiaron del evento. En menor medida, también se aprovecharon los comercios y los locales de ocio. Concretamente, el gasto medio en alojamiento fue de $119,6 €$ por persona, mientras que el gasto en manutención fue de 93,6 $€$ por persona.

Lo anteriormente señalado pone de manifiesto una circunstancia que no se debería perder de vista cuando se procede con un estudio de impacto económico como el aquí realizado. Los datos obtenidos resultan incuestionables al no estar valorando efectos de difícil cuantificación o medición (como los intangibles o subjetivos) pero también es cierto que el reparto no es homogéneo para todos los sectores económicos. Quiere esto decir que de manera directa los beneficiados son los sectores más relacionados con el terreno turístico y solo de manera indirecta este efecto económico alcanza a sectores económicos más alejados.

Desde el punto de vista turístico, la percepción es ligeramente inferior cuando se pregunta por Pontevedra como lugar de vacaciones, el cuál tiene que ver con una serie de limitaciones que es posible extraer de los datos de las encuestas realizadas. El casco viejo se muestra como el lugar más atractivo de Pontevedra, mientras que la zona de la planta de celulosa es considerada por los asistentes como la que menos les gusta.

Así, el aspecto más criticado de la instalación fue la falta de aparcamiento (en ocasiones se vio agravado con la presencia de la grúa algún día de la competición retirando bastantes vehículos con el consiguiente malestar de los damnificados). Esto está claramente relacionado con la insuficiente dotación en materia de transporte urbano (conexiones entre la ciudad y la piscina) a las que se le puede añadir también la deficiente conexión con los aeropuertos (especialmente el de Vigo a escasos 25 minutos de la instalación).

Otra aspecto comentado negativamente fue la escasez de oferta de restauración próxima a la piscina y, de manera particular, en la propia piscina. 


\section{Referencias bibliográficas}

1. Barajas, A., Salgado, J. y Sánchez, P. (2012). Problemática de los estudios de impacto económico de eventos deportivos. Estudios de Economía Aplicada, 30 (2), 441-462.

2. Burns, J. P. A., Hatch, J. H. y Mules, T. J. (1986). The Adelaide Grand Prix: The impact of a special event. The Centre for South Australian Economic Studies, Adelaide.

3. Fernández, M.T. (2014). El impacto turístico de los eventos deportivos: un estudio de caso. Cuadernos de turismo, 33, 59-76.

4. Goldman, M. y Johns, K. (2009). Sportainment: changing the pace of limited-over cricket in South Africa. Management Decision, 47 (1), 124 136.

5. Henderson, J.C., Foo, K., Lim, H. y Yip, S. (2010). Sports events and tourism: the Singapure Formula One Grand Prix. International Journal of Event and Festival Management, 1.3, 60-73.

6. Latiesa, M. y Paniza, J.L. (2006). Turistas deportivos. Una perspectiva de análisis. Revista Internacional de Sociología, LXIV (44), mayo-agosto, 133-149.

7. Leal Rojas, C. L. (2005). Valoración económica del medio ambiente: Caso de la reserva de la biosfera de los tuxtlas. Tesis Licenciatura. Departamento de Economía, Escuela de Ciencias Sociales, Universidad de las Américas.

8. Lera, F. (2010). Una aproximación al Deporte desde la Economía. El Papel de la Fundación Observatorio Económico del Deporte (FOED). Encuentro Nacional de Observatorios del Deporte, 3-5 Noviembre 2010, Sevilla.

9. Magaz-González, A.M. y Fanjul-Suárez, J.L. (2012). Organización de eventos deportivos y gestión de proyectos: factores, fases y áreas. Revista Internacional de Medicina y Ciencias de la Actividad Física y el Deporte, 12 (45), 138-169.

10. Parent, M.M \& Seguin, B. (2007). Factors That Led to the Drowning of a World Championship Organizing. Committee: A Stakeholder Approach. European Sport Management Quarterly, Routledge.
11. Póveda Andrés, J. L., Hernández Sansalvador, M., Díez Martínez, A. M., y García Gómez, C. (2004). Análisis coste-beneficio del proceso de semiautomatización en la preparación de dosis unitarias por el servicio de farmacia. Farmacia Hospitalaria, 28 (2), 76-83.

12. Ramírez J.M., Ordaz, J.A. y Rueda, J.M (2007). Evaluación del impacto económico y social de la celebración de grandes eventos deportivos a nivel local: el caso del Campeonato de Tenis femenino de la ITF en Sevilla en 2006 Evaluación del impacto económico y social de la celebración de grandes eventos deportivos a nivel local: el caso del Campeonato de Tenis femenino de la ITF en Sevilla en 2006, Revista de métodos cuantitativos para la economía y la empresa, 20-39.

13. Rodríguez Guerrero, P., Pujol, F. y Baños, J. (2011). Análisis del impacto económico de la celebración del campeonato del mundo de patinaje de velocidad (CMPV) celebrado en Gijón. 4-12 septiembre de 2008. Fundación Observatorio Económico del Deporte.

14. Sánchez, P.; Barajas, A. y Alén, E. (2013). Los eventos deportivos como herramienta de promoción turística. Revista de Análisis Turístico, 16, 59-69.

15. Shone, A. y Parry, B. (2001). Successful event management: a practical handbook. London. Continuum.

16. Sport England (1999). Measuring Success 2: The Economic Impact of Major Sports Events. Londres.

17. Velasco, M. y Rodríguez-Monguió, R. (2009). Análisis coste-beneficio: Metodología y utilidad en la toma de decisiones sanitarias. Investigación Centro de Estudios en Economía de la Salud y de la Politica Social.

18. Walker, S. y Enz, M. (2006). The impact of professional sports on the local economy. Western New England Law Review, 29, 149-163.

19. Walo, M., Bull, A. y Breen, H. (1996). Achieving economic benefits at local events: a case study of a local sports event. Festival Management \& Event Tourism, 4, 95-106. 